\title{
La desmotivación y su relación con factores académicos y psicosociales de estudiantes universitarios
}

\author{
Isolina González Castro ${ }^{1}$; Mario Alberto Vázquez García ${ }^{2}$; Martha Alejandrina Zavala Guirado*3 \\ ${ }^{1}$ https://orcid.org/0000-0002-7265-6725, Instituto Tecnológico de Sonora Unidad Guaymas, Sonora, México, ${ }^{2}$ https://orcid. \\ org/0000-0002-6490-838X, Instituto Tecnológico de Sonora Unidad Guaymas, Sonora, México, ${ }^{3}$ https://orcid.org/0000-0001-9177- \\ 0411, Instituto Tecnológico de Sonora Unidad Guaymas, Sonora, México
}

Citar como: González, I., Vázquez, M. \&, Zavala, M. (2021). La desmotivación y su relación con factores académicos y psicosociales de estudiantes universitarios. Revista Digital de Investigación en Docencia Universitaria, 15(2), e1392. https://doi. org/10.19083/10.19083/ridu.2021.1392

Recibido: 08/01/2021. Revisado: 01/03/2021. Aceptado: 15/10/2021. Publicado: 26/11/2021.

\section{Resumen}

Introducción: La deserción escolar constituye una problemática entre las instituciones de educación superior, que prevalece y afecta el desarrollo profesional de los estudiantes. Objetivo: Comprobar relaciones entre desmotivación, competencias docentes, satisfacción de expectativas académicas y tutorías en una universidad del sur de Sonora, México. Método: Se empleó una escala de factores asociados a la deserción (Escala de Tutoría, Escala de Satisfacción de Expectativas Académicas, Escala de Competencias Docentes y Escala de Motivación), aplicada a 359 estudiantes de seis carreras, elegidos al azar, 161 son hombres, 191 mujeres, con edades de entre 18 a 51 años. Resultados: Indican que la tutoría, satisfacción de expectativas académicas y competencias docentes tuvieron covarianzas positivas y significativas. Con respecto a la desmotivación, esta covarió de manera negativa con los tres constructos restantes, lo que sugiere validez divergente entre el resto de los constructos. Conclusión: A mayores niveles de tutoría, satisfacción de expectativas académicas y competencias docentes, existirán menores niveles de desmotivación. Se discuten las limitantes del estudio.

Palabras clave: calidad de la educación; educación y desarrollo; innovación pedagógica; motivación; tutoría (educación).

\section{Demotivation and its relation with academic and psychosocial factors in university students}

\begin{abstract}
Introduction: School dropout represents a problem among higher education institutions, that prevails and affects the professional development of students. Objective: Prove relations between demotivation, teaching skills, satisfaction of academic expectations and tutoring in a University in a South of Sonora, México. Method: A scale of factors associated with dropping out was used (Tutoring Scale, Scale of Satisfaction of Academic Expectations, Scale of Teaching Competencies and Scale of Motivation), applied to 359 students from 6 different professions, chosen at random where 161 were men, 191 women, aged from 18 to 51 years old. Results: Tutoring, academic expectations satisfaction and teaching competences had a positive and significative covariances among these constructs. Regarding the demotivation factor, it was appreciated a
\end{abstract}


negative covariance with the remaining constructs, which suggest the presence of divergent validity between this and the rest of the constructs. Conclusion: At higher levels of tutoring, academic and teaching competences expectations satisfaction, there will be lower levels of demotivation. The limitations of the study are discussed.

Key words: quality of education; education and development; pedagogical innovation; motivation; tutoring (education).

\section{Introducción}

La deserción escolar constituye una gran preocupación dentro de las instituciones de educación superior, ya que por más esfuerzos que se realicen por solucionarla con financiamientos, programas educativos de calidad y fortalecimiento de la capacitación docente, el fenómeno prevalece y afecta el desarrollo profesional de los estudiantes, por lo que se ha convertido en objeto de estudio a fin de encontrar variables relacionadas o causantes del abandono de los estudios.

Al indagar sobre cuáles son aquellos factores que propician la deserción en estudiantes universitarios se han generado modelos y teorías que intentan explicar el fenómeno. Zavala et al. (2015) elaboraron un modelo organizando los factores externos, internos y bilaterales, y encontraron que la falta de motivación es uno de los elementos que constituye los aspectos internos del alumno que provocan su deseo por abandonar los estudios.

En otro estudio llevado a cabo en México, Mares et al. (2012) realizaron una correlación entre las características motivacionales de los estudiantes y sus competencias académicas, corroborando que las primeras resultan ser un factor de mayor impacto, que puede llevar a los estudiantes a tomar la decisión por desertar.

Por otro lado, en una investigación, Freixa et al. (2018) concluyeron que la deserción universitaria es consecuencia de múltiples factores entre los que destacan aspectos del contexto que pueden influir en la decisión por desertar, así como factores psicosociales o de índole académico, como por ejemplo que no estén contentos con la carrera que eligieron, o con los logros en sus cursos e incluso las características individuales de cada uno. Según los antecedentes los factores que se analizarán en el presente estudio son la motivación, la satisfacción de expectativas académicas, la tutoría y el desempeño docente.

De acuerdo con Ramírez y Olmos (2020) la motivación en un contexto educativo se puede definir como la actitud positiva para que se logre el aprendizaje. Desde el punto de vista de Naranjo (2010), la motivación puede atender a factores personales o académicos, siendo la actitud del docente uno de los factores académicos que provoca la desmotivación por parte de los estudiantes. En ese sentido, Rodríguez-Pérez (2012) y Triana et al. (2016) resaltaron el papel tan determinante que juega la motivación en los estudiantes a través del papel del docente, la metodología que utilice y las relaciones que establezca para promover la interacción entre él y los otros estudiantes, logrando modificar el interés, las necesidades, incluso las habilidades de los estudiantes.

La motivación destaca un especial interés por los aspectos cognoscitivos, la inteligencia o el aprendizaje significativo y resolver situaciones motivacionales en ámbitos diversos como el social, educativo (Armas, 2019). Esta diversificación permite el surgimiento de puntos de vista diversos que ayudan en la comprensión del constructo de motivación en áreas determinadas (Reeve, 2008).

Según la teoría de la autodeterminación, la motivación no tiene un solo componente que la defina, está compuesta por tres aspectos o enfoques de análisis; el global, el contextual y el situacional. El global tiene una orientación general y se asocia con la personalidad del sujeto. El contextual ubica al sujeto en actividades específicas como la educación o el trabajo, siendo influenciado en forma directa por aspectos de tipo social. Con relación al ámbito situacional, se ubican acciones o situaciones específicas en un momento preciso y que no volverán a presentarse en su vida (Stover et al., 2017).

Por otro lado, según Pichardo et al. (2007), las expectativas se han estudiado en psicología $\mathrm{y}$ educación bajo el precepto de las influencias de unas personas sobre otras y que las expectativas mismas llegan a convertirse en realidad, lo que se constituye en un proceso en el que las creencias y las expectativas afectan su comportamiento que lleva a los demás a una respuesta que confirma 
esas creencias. Este fenómeno ha sido denominado el efecto Pigmalión o profecía de autocumplimiento. Del mismo modo, Bandura (1977) para describir los elementos de las expectativas incluyó en sus términos el alcance de metas basándose en las experiencias de los individuos, así como las expectativas de resultados, las expectativas de eficacia. Para el análisis de las expectativas de eficacia personal consideró también cuatro elementos; como logros de desempeño, experiencias indirectas, persuasión verbal y estados fisiológicos, con las que al final el individuo manifestará o no comportamientos de afrontamiento sobre diferentes experiencias.

Las expectativas han sido estudiadas tanto desde el punto de vista de los profesores como de los estudiantes. En lo que se refiere a estos últimos, las investigaciones han intentado determinar lo que esperan de la universidad como institución y sus servicios; también se han analizado las expectativas que tienen hacia el proceso de enseñanza-aprendizaje, siendo en esta parte donde se ha examinado la relación del docente con las expectativas (Castañeda \& Alarcón, 2019; Pérez, 2015; Pichardo et al., 2007). Están representadas por todo aquello que los estudiantes esperan hacer y los logros en la universidad, simbolizadas en las dimensiones académicas y de ajuste social. Pueden servir de orientación cognitiva y de motivación; además, ayudan a que los estudiantes se comprometan con su educación, por lo que se hace necesario que las instituciones conozcan qué esperan los estudiantes sobre su satisfacción y necesidades para realizar planeaciones que las atiendan (Conde et al., 2017).

En las opiniones de Orozco et al. (2021) y Torres et al. (2014), las competencias docentes hacen referencia a todo aquello que guarda relación con la práctica docente, con sus fines, su mejora y profesionalización. Además, argumentaron que en ellas se integran saberes, habilidades y actitudes relacionados con la investigación, la vinculación, la difusión, la calidad de la docencia, la cooperación y el liderazgo en diferentes contextos educativos, además las competencias que debería desarrollar un docente son la planeación y organización del aprendizaje, gestionar el aprendizaje, comunicar de manera efectiva, valorar el aprendizaje alcanzado, y organizar su actualización y formación.
En el mismo sentido, la Organización de las Naciones Unidas para la Educación, la Ciencia y la Cultura y la Organización Internacional del Trabajo (Unesco \& OIT, 2008) establecieron como deberes de los docentes universitarios enseñar de manera eficaz, ser imparciales y equitativos con los estudiantes y colegas, fomentar el intercambio de ideas de manera libre, realizar investigaciones de manera ética y difundir sus conclusiones o mantenerse actualizado sobre la materia en la que se está especializando; además de administrar de manera honrada los fondos que se le asignen. Asimismo, Salazar et al. (2016) señalaron que en el perfil profesional del docente las competencias estarían referidas a: (a) el saber, explicado por los conocimientos, información, teorías; (b) saber hacer, al realizar acciones competentes; y (c) saber ser y saber estar, explicado a través de valores y las normas que requiere el desempeño.

Arias et al. (2018), considerando las recomendaciones de la Unesco y la Organización para la Cooperación y el Desarrollo Económicos (OCDE), establecieron que las competencias docentes universitarias se componen de destrezas, habilidades, aptitudes, actitudes y valores para la enseñanza y el aprendizaje con el fin de que los estudiantes desarrollen sus competencias para su vida profesional. Algunas de las competencias docentes universitarias son selección de contenidos, darse a comprender, uso de TIC, diseño y organización de actividades para el aprendizaje, comunicación con alumnos, tutorías, evaluación de la docencia y del aprendizaje, investigación, trabajo en equipo, y uso de técnicas didácticas.

Con relación al efecto de las competencias de los profesores universitarios, Díaz y Osuna (2021), y Román (2013) encontraron que tienen un fuerte impacto en la permanencia o en la deserción de los estudiantes, lo cual puede determinarse con la interacción, la implementación de ciertas prácticas pedagógicas, al ofrecer estrategias cognitivas y socioafectivas, al propiciar el desarrollo de habilidades y capacidades, además al mostrar interés, motivación, ciertas expectativas o actitudes, siendo el buen desempeño docente un factor protector del abandono o el fracaso escolar.

Dentro de las prácticas pedagógicas se encuentra la tutoría, definida como la acción pedagógica 
con la que se da seguimiento, acompañamiento o guía en procesos educativos, para el mejoramiento de las trayectorias de los estudiantes partiendo de sus necesidades y condiciones, además de pretender su formación integral (Cambours de Donini et al., 2011; Martínez et al., 2017). También es considerada como una acción docente de intervención con fines de formación establecida para el seguimiento de los estudiantes y que estos desarrollen cualidades racionales (Aguilera, 2019; Arbizu et al., 2005). El concepto aun cuando ha sido abordado en diferentes lenguajes, en inglés mentoring, tutoring, coaching, o en francés tutorat, conseil pedagogique, acompagnement, poseen algo en común, un profesor-tutor que cumple las funciones de guía u orientación en aspectos académicos, administrativos e intelectuales en los estudiantes (Delgado-García et al., 2020; Lobato et al., 2004).

A través de la acción tutorial se pueden atender factores relacionados con la integración a la vida universitaria, desarrollo académico, definición del perfil profesional, el desarrollo de competencias, la inserción al mundo productivo y el desarrollo personal (Pérez et al., 2017). Además, son una estrategia de prevención, el tutorado es orientado en su desarrollo académico, personal y profesional, también es un soporte para el aprendizaje, y se facilita la adquisición de ciertas habilidades (De la Cruz et al., 2011; Martínez et al., 2016).

Es determinante estudiar los factores relacionados con la deserción en la educación superior, pues representa un problema grave que afecta a la mayoría de las IES, en esta universidad del sur de Sonora, objeto de estudio, los porcentajes de deserción que maneja cada programa educativo, según información proporcionada por el Departamento de Registro Escolar, específicamente en la cohorte correspondiente al 2009 en un campus, en donde Ingeniería Industrial y de Sistemas (IIS) registró una deserción total del 42.5\%, Ingeniería en software (ISW) un 16.6\%, Licenciado en Administración (LA) y Licenciado en Administración de Empresas Turísticas (LAET) ambas con un 49.9\%, Licenciado en Ciencias de la Educación (LCE) tuvo una tasa de deserción del 24.3\% y el programa de Licenciado en Psicología aunque aún no cuenta con una generación egresada presenta un $36 \%$ de deserción. Y en otro de los cam- pus el 33.33\% desertó de IIS, el 26\% de LA, el 30\% de LCE y el 35\% de LAET. En términos generales, se puede determinar que el $35.25 \%$ y el 31.37 respectivamente de los estudiantes inscritos en algunos de los programas educativos de la cohorte 2009 en estos campus desertó (Instituto Tecnológico de Sonora [ITSON], 2015).

Considerando lo anterior, este estudio identifica los factores psicosociales del estudiante como son la falta de motivación y la satisfacción de expectativas académicas; los factores institucionales y administrativos referidos a tutoría y las competencias docentes. El estudio plantea el objetivo de comprobar las relaciones entre estos cuatro constructos en una universidad del sur de Sonora (México). Para ello, tres hipótesis fueron propuestas:

1. La tutoría se relaciona con la Satisfacción de Expectativas Académicas y las Competencias Docentes.

2. Las Competencias Docentes se relacionan con la Satisfacción de Expectativas Académicas.

3. La Desmotivación se asocia negativa y significativamente con la Tutoría, las Satisfacción de Expectativas Académicas y las Competencias Docentes.

\section{Método}

\section{Diseño}

El enfoque de la investigación es cuantitativo y su alcance es explicativo, se busca una explicación y determinación de los fenómenos (Hernández et al., 2014). En el contexto cuantitativo se pueden aplicar estudios de tipo predictivo en los que se establezca una relación causal entre diversas variables se pretende comprobar la relación e influencia de variables cuantitativas y establecer un modelo de ajuste predictivo.

\section{Participantes}

Para la realización del estudio se utilizó el método probabilístico Hernández et al. (2014), con alumnos inscritos en seis programas educativos, seleccionados de forma aleatoria, todos estudiantes de una universidad del sur de Sonora, siendo un 
total de 359 alumnos que representan el $40 \%$ de la población estudiantil, de diversos semestres, de los cuales 161 son hombres (45\%), 191 mujeres (53\%) y ocho valores perdidos (2.2\%), con edades entre los 18 hasta los 51 años, 351 de ellos reportaron estar solteros, mientras que siete casos indicaron estar casados. Respecto del ingreso familiar se apreció que se ubican en la categoría de 2,501 a 4,000 pesos mensuales.

\section{Instrumento}

Para el desarrollo del estudio se utilizó una escala de factores asociados a la deserción elaborada por Zavala, et al. (2018), el instrumento mostró índices altos de consistencia interna por cada factor (el coeficiente Omega varió entre .89 y .95 para los doce factores). Se obtuvo un modelo confirmatorio con una aceptable bondad de ajuste (Chi cuadrado = 151.94; $\mathrm{P}=0.00001 ; \mathrm{CFI}=.91$; RMSEA = .089; intervalo de confianza al 90\% de RMSEA = .07, .10 y coeficiente $r_{s}=.86$, donde se seleccionaron cuatro constructos: Tutoría, Competencias docentes, Satisfacción de expectativas y Desmotivación.

\section{Escala de Tutoría}

Evalúa aspectos relacionados con la solución de problemas académicos de los estudiantes, así como la promoción de estrategias para el estudio. Posee seis reactivos, en formato de respuesta $\mathrm{Li}$ kert con cinco opciones de respuesta ( 1 = Muy en Desacuerdo hasta 5 = Totalmente de Acuerdo). Un ejemplo de reactivo es "el tutor mostró interés en los problemas académicos que tuviste".

\section{Escala de Competencias Docentes}

Se utilizó para medir aquellas actividades que desarrollan los docentes durante la impartición de clases, así como evaluar actitudes de interacción positiva hacia los estudiantes. Cuenta con nueve reactivos, con cinco opciones de respuesta en formato Likert ( $1=$ Nunca hasta $5=$ Siempre). Un reactivo que ejemplifica esta escala es "los docentes emplean diferentes estrategias para enseñar".

\section{Escala de Satisfacción de Expectativas cadémicas}

Evalúa el grado de identificación del estudiante en relación con la universidad, la carrera elegida y lo que espera de cada una de ellas. Se integra por cuatro reactivos, con cinco opciones de respuesta en formato Likert ( 1 = Definitivamente no hasta $5=$ Definitivamente sí). Ejemplo de un reactivo de esta escala es "te resulta interesante la carrera profesional que elegiste".

\section{Escala de Desmotivación}

Enfoca sus indicadores hacia el desempeño académico en la obtención de calificaciones aprobatorias, así como el esfuerzo por asistir y llegar a tiempo a clases. Posee seis reactivos, en formato Likert, con cinco opciones de respuesta ( $1=$ Definitivamente no hasta 5 = Definitivamente sí). Un reactivo para ejemplificar el contenido de esta escala es "Te esfuerzas por superarte en el estudio". Cabe señalar que las respuestas de los reactivos fueron recodificadas inversamente.

\section{Procedimiento}

Para la realización del estudio se solicitó la autorización de los estudiantes con la firma de un consentimiento informado en la que expresaban estar de acuerdo en participar en el estudio, se les dio a conocer sus derechos y responsabilidad ética en las respuestas que emitieran y se les aseguró la protección de la identidad de las personas que participan como informantes de la investigación. Posteriormente se visitó a los grupos aplicándose el cuestionario de manera grupal a 359 estudiantes universitarios, en los semestres de segundo, cuarto y sexto, sin embargo, dentro de esos grupos también se aplicó a alumnos de primero, tercero, quinto, séptimo, octavo y décimo semestre, con un tiempo de respuesta entre los 20 y 30 minutos.

\section{Análisis de datos}

Para el análisis se seleccionaron las escalas del instrumento, se procedió a computar estadísticas descriptivas con el programa estadístico SPSS versión 27.0, para determinar el coeficiente Omega de McDonalds se utilizó el programa JASP para cada indicador, y el software EQS para el modelo de covarianzas. A partir de variables indicadores constituidas por las medias de los reactivos de cada factor, se procedió a efectuar la prueba de correlaciones bivariadas de Pearson, para evidenciar la relación entre 
los cuatro constructos abordados, como paso previo a la prueba del modelo de covarianzas.

Antes de analizar los resultados de las covarianzas y asegurar la posibilidad de relaciones causales se deben tener indicios de validez convergente si existen pesos factoriales altos y significativos entre las variables observadas y los factores propuestos a los que corresponden (Kline, 2005; Nunnally \& Bernstein, 1995). En cuanto a la validez divergente, se obtiene indicios de esta si dos constructos distintos exhiben correlaciones negativas (Campbell \& Fiske, 1959).

Asimismo, se observó si los datos satisfacían el supuesto de normalidad multivariada; en caso contrario, se sugiere estimar el modelo con el método robusto de Máxima Verosimilitud (Satorra \& Bentler, 2001). Autores como West et al. (1995) sugirieron evaluar si el valor del coeficiente normalizado multivariante de Mardia se sitúa en valores superiores a siete, lo que señalaría no normalidad multivariada. Para evaluar si el modelo de covarianzas entre los cuatro constructos exhibe bondad de ajuste, se consideraron seis indicadores. Se evaluó si el valor de $\chi 2$ era no significativo ( $p$ $>$.05), lo cual indica la ausencia de discrepancia entre la matriz de datos con el modelo propuesto. Sobre los índices prácticos de ajuste, se valoraron el CFI, el NNFI y el RMSEA. De los dos primeros se tiene como criterio que sus valores se ubiquen por arriba de .95, mientras que del tercero se sugiere que sea menor a .06 (Hu \& Bentler, 2009). Finalmente, el quinto indicador por evaluar consistió en el intervalo de confianza de 90\% del RMSEA, del cual se sugiere su límite superior no rebase la cifra de .10 (Kline, 2005).

\section{Resultados}

Se obtuvieron estadísticas descriptivas de los reactivos que integraron cada escala y se computó su correspondiente coeficiente Omega por ser considerada una medida adecuada de confiabilidad y permite una medida más precisa. Los resultados se exhiben en la Tabla 1.

Los descriptivos en tutorías demuestran que los estudiantes están ni de acuerdo ni en des- acuerdo en los apoyos y acompañamiento de los docentes en lo que concierne al programa de tutorías, sin embargo, con relación a las competencias docentes manifestaron que casi siempre muestran actitudes de interacción positiva hacia los estudiantes, motivan y emplean estrategias relevantes para ellos. En cuanto a la satisfacción de expectativas se encontró que definitivamente sí han cumplido con lo deseado y hay sentido de pertenencia hacia su universidad y sobre la desmotivación no se encontraron indicadores negativos en su desempeño académico en la obtención de calificaciones aprobatorias, así como el esfuerzo por la asistencia y puntualidad. Los cuatro factores analizados presentaron alta fiabilidad, lo que demuestra la confianza en los resultados de la escala.

Se llevó a cabo un análisis de correlaciones bivariadas de Pearson para conocer el grado de asociación, en términos de dirección y magnitud, que presentaban los constructos abordados. Los resultados pueden apreciarse en la Tabla 2.

Según los resultados hay correlación negativa con el constructo desmotivación y el resto, esto indica que cuando hay desmotivación se mueve en relación opuesta a la tutoría, competencias docentes y la satisfacción de expectativas. El resto de las correlaciones son positivas y significativas demostrando que estas variables se mueven en la misma dirección.

Tomando en cuenta los resultados del análisis de correlación, se procedió a la prueba de un modelo estructural de covarianzas entre los constructos. El valor del coeficiente normalizado de Mardia se situó en 43.56, evidenciando no normalidad multivariada. Por lo tanto, la estimación del modelo se realizó con el método Robusto de Máxima Verosimilitud. Respecto de los valores de los indicadores de ajuste, se obtuvo que la prueba Chi cuadrado de Satorra-Bentler (S-B $\chi 2)$ fue de 425.12, con 269 grados de libertad, siendo significativa $(\mathrm{p}<.05)$. No obstante, los índices prácticos revelan evidencia de ajuste: el CFI alcanzó el valor de .95, el NNFI exhibió la cifra de .94, mientras que el RMSEA obtenido quedó en .04. Asimismo, el intervalo del 90\% del RMSEA tuvo valores adecuados (.03 y .05). Todos los pesos factoriales obtuvieron valores significativos $(t>1.96, p<.05)$. 
Tabla 1

Estadísticas descriptivas de las medidas empleadas

\begin{tabular}{|c|c|c|c|c|c|}
\hline Escala / Reactivos & Min & Max & $\mathbf{M}$ & DT & $\omega$ \\
\hline \multicolumn{6}{|l|}{ Tutoría } \\
\hline $\begin{array}{l}\text { Tu integración a la universidad ha mejorado con el programa de tutorías } \\
\text { académicas. }\end{array}$ & 1 & 5 & 3.42 & 1.15 & 0.89 \\
\hline El tutor te orientó sobre hábitos de estudio. & 1 & 5 & 3.61 & 1.17 & 0.89 \\
\hline El tutor te recomendó técnicas para que pudieras estudiar con eficacia. & 1 & 5 & 3.45 & 1.23 & 0.89 \\
\hline $\begin{array}{l}\text { El tutor te ayudó a buscar soluciones para los problemas que presentaste durante } \\
\text { la asistencia a las tutorías. }\end{array}$ & 1 & 5 & 3.63 & 1.20 & 0.89 \\
\hline El tutor te apoyó en las actividades a realizar para la solución de problemas. & 1 & 5 & 3.58 & 1.18 & 0.89 \\
\hline El tutor mostró interés en los problemas académicos que mostraste. & 1 & 5 & 3.88 & 1.11 & 0.89 \\
\hline \multicolumn{6}{|l|}{ Competencias docentes } \\
\hline Los docentes siguen el programa de curso. & 1 & 5 & 4.36 & .711 & 0.89 \\
\hline Los docentes establecen las reglas considerando a los alumnos. & 1 & 5 & 4.09 & .873 & 0.89 \\
\hline Los docentes te motivan para realizar las actividades y tareas en clase. & 1 & 5 & 3.98 & .931 & 0.89 \\
\hline Los docentes brindan asesorías cuando se les solicita. & 1 & 5 & 3.96 & 1.00 & 0.89 \\
\hline Los docentes emplean diferentes estrategias para enseñar. & 1 & 5 & 4.12 & .867 & 0.89 \\
\hline Los docentes proporcionan actividades relevantes a los alumnos. & 1 & 5 & 4.05 & .847 & 0.89 \\
\hline Los docentes retroalimentan las tareas asignadas. & 1 & 5 & 4.12 & .790 & 0.89 \\
\hline Los docentes apoyan a los alumnos y los ayudan a resolver problemas académicos. & 1 & 5 & 4.06 & .864 & 0.89 \\
\hline Los docentes evalúan y retroalimentan en cada parcial. & 1 & 5 & 4.14 & .902 & 0.89 \\
\hline \multicolumn{6}{|l|}{ Satisfacción de expectativas } \\
\hline Hasta el momento la carrera ha cumplido con tus expectativas. & 1 & 5 & 4.18 & .932 & 0.89 \\
\hline Te sientes identificado con la universidad. & 1 & 5 & 4.15 & .978 & 0.89 \\
\hline Te resulta interesante la carrera profesional que elegiste. & 1 & 5 & 4.57 & .805 & 0.89 \\
\hline Te sientes orgulloso de pertenecer a la universidad. & 1 & 5 & 4.33 & .951 & 0.89 \\
\hline \multicolumn{6}{|l|}{ Desmotivación } \\
\hline Te esfuerzas por superarte en el estudio ( $R$ ). & 1 & 5 & 1.38 & .613 & 0.89 \\
\hline Te esfuerzas por obtener buenas calificaciones en todas las materias (R). & 1 & 5 & 1.51 & .783 & 0.90 \\
\hline Eres puntual al asistir a clases (R). & 1 & 5 & 1.73 & .884 & 0.90 \\
\hline Asistes a clase con normalidad en los horarios establecidos ( $R$ ). & 1 & 5 & 1.42 & .723 & 0.90 \\
\hline Hasta el momento tienes acreditadas las asignaturas que has cursado (R). & 1 & 5 & 1.56 & .927 & 0.90 \\
\hline Consideras que tienes un promedio mayor de 8 (R). & 1 & 5 & 1.65 & 1.02 & 0.90 \\
\hline
\end{tabular}

Tabla 2

Correlaciones bivariadas de Pearson

\begin{tabular}{lllllll}
\hline Constructos & $\mathbf{M}$ & $\mathbf{D E}$ & $\mathbf{1}$ & $\mathbf{2}$ & $\mathbf{3}$ & $\mathbf{4}$ \\
\hline 1 Tutoría & 3.60 & 1.03 & 1.0 & & & \\
2 Competencias docentes & 4.10 & .609 & $.44^{*}$ & 1.0 & \\
3 Satisfacción de expectativas académicas & 4.30 & .757 & $.29^{* *}$ & .40 & 1.0 & \\
4 Desmotivación & 1.54 & .582 & $-.16^{* *}$ & -.24 & -.31 & 1.0 \\
\hline
\end{tabular}




\section{Figura 1}

Modelo de covarianzas entre tutoría, competencias docentes, satisfacción de experiencias académicas y desmotivación pesos factoriales y covarianzas significativas

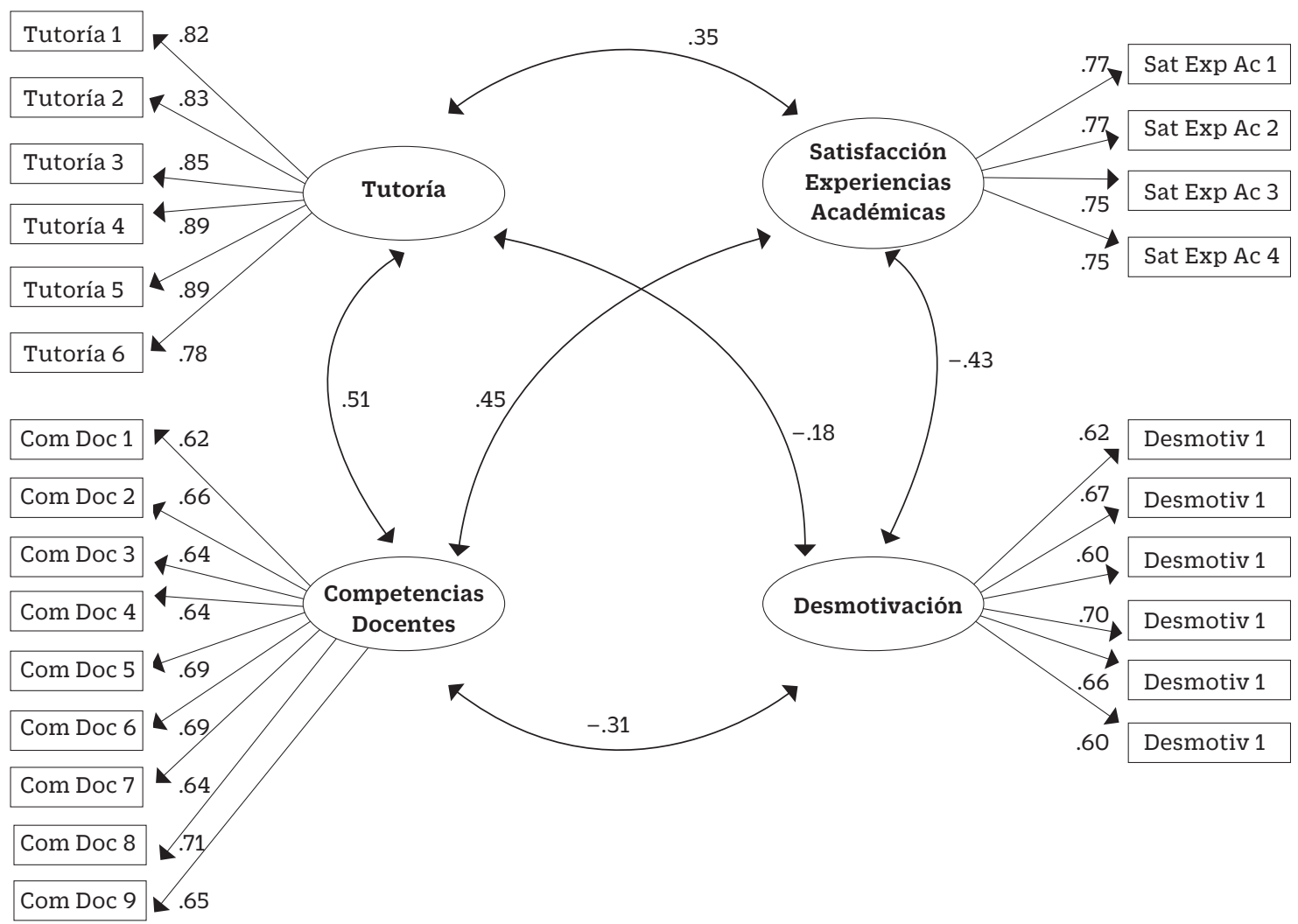

Nota. $(t>1.96, p<.05)$. Bondad de Ajuste: $S-B \chi 2(269$ grados de libertad, $N=359)=425.12, p>.05 ; C F I=.95$; $N N F I=.94 ;$ RMSEA $=.04$. Intervalo de Confianza del $90 \%$ del RMSEA $(.03, .05)$

Se apreció que entre tres de los cuatro constructos abordados (Tutoría, Satisfacción de Expectativas Académicas y Competencias Docentes) hubo covarianzas positivas y significativas directamente proporcionales una respecto de la otra, mostraron que varían en la misma dirección. Por otro lado, el factor de Desmotivación se apreció que este covarió de manera negativa con los tres constructos restantes, lo cual sugiere cuando una variable crece la otra variable decrece. De acuerdo con los resultados, ante mayor incremento de Tutoría, Satisfacción de Expectativas Académicas y Competencias Docentes, será mayor el decrecimiento de Desmotivación, lo cual se encuentra respaldado por la literatura especializada.

\section{Discusión}

En relación con la primera hipótesis, que se refiere a las relaciones de la tutoría con la Satisfacción de Expectativas Académicas y las Competencias Docentes, se halló evidencia que la confirma. Con las acciones de tutoría se puede mejorar el rendimiento académico de los estudiantes, incluidas aquí las materias aprobadas por semestre (Cuevas et al., 2017; Guerra-Martín et al., 2017), también se puede maximizar el potencial académico de los alumnos al exponerse a la tutoría (Walvoord \& Pleitz, 2016). Del mismo modo, los estudiantes pueden ganar confianza, sentirse menos intimidados ante ejercicios de revisión y exámenes, 
trabajar de manera colaborativa al participar de manera voluntaria en las tutorías (Eaton, 2015). Además, se puede influir de manera positiva en la adquisición de ciertas habilidades, la percepción de la autoeficacia y las actitudes hacia la acción tutorial (Uzuner \& Aktas, 2016). Por último, pueden desarrollar habilidades de autorregulación cognitiva como el monitoreo, la evaluación y la orientación (De Backer et al., 2014).

Considerando la segunda hipótesis, que postulaba que las Competencias Docentes se relacionan con la Satisfacción de Expectativas Académicas, también se confirmó. Según Feldman y Theiss (1980), las expectativas de los estudiantes y los profesores afectan sus actitudes y comportamientos, además de las personas con las que interactúan. Estos autores resaltan que de manera simultánea pueden mantener sus expectativas de tal forma que afectarán sus actitudes sobre ellos mismos, sus pares y la situación de enseñanza en general. Asimismo, cuando los estudiantes esperan encontrar en el aula a un profesor competente, lo perciben de manera positiva, también a la lección; sucede lo contrario cuando tienen la expectativa de que será un profesor deficiente. Los autores coincidieron con otros investigadores sobre el hecho de que los estudiantes al esperar un profesor deficiente, tienden a presentar actitudes y comportamientos menos favorables sobre el mismo profesor y su propio aprendizaje.

Según Villalobos et al. (2010), conocer las percepciones y expectativas que tienen los estudiantes universitarios sobre la docencia universitaria ayuda a promover un cambio en las estructuras y prácticas pedagógicas de la enseñanza superior. Los datos obtenidos revelan la necesidad de revisar la actual concepción que se tiene de la docencia universitaria, sobre todo en lo referente a la educación de pregrado, en donde los alumnos reportan una percepción menos positiva de la docencia y, lo que requiere de mayor atención, menores expectativas. En este mismo sentido, Morgan (2015) estableció que las expectativas y las experiencias de otros estudios pueden ser útiles para captar más alumnos al mejorar los procesos educativos, incrementar las tasas de retención y las experiencias de los estudiantes.

En cuanto a la última hipótesis, la cual estable- cía que la desmotivación se asocia negativa y significativamente con la tutoría, la satisfacción de expectativas académicas y las competencias docentes, también se corroboró: hubo covarianzas negativas entre la desmotivación y los tres constructos anteriores. Según Freixa et al., (2018), para estudiantes que tienen que trabajar y estudiar al mismo tiempo, implicando un doble esfuerzo por mantenerse en la universidad, la insatisfacción de las expectativas académicas y la desmotivación llegan a ser factores determinantes para que abandonen sus estudios.

Según Torres (2016), ya no basta con tener en las aulas a docentes con sus planeaciones, metodologías, materiales listos para el buen desarrollo de una clase, lo que realmente buscan los estudiantes es el afecto que permita establecer una clase llena de confianza, donde el docente se relacione con los alumnos, el establecimiento de un ambiente de seguridad, de respeto. El acompañamiento, el conocer las necesidades y expectativas de los alumnos, además el contar con la habilidad para provocar en ellos el deseo por continuar con su proceso de formación hasta la culminación exitosa de sus estudios, son elementos que actualmente se requieren para mantener la motivación del estudiantado. Finalmente, el aspecto afectivo promovido por el docente podrá provocar la motivación o desmotivación en los estudiantes.

Se concluye que con las acciones de tutoría se puede mejorar el rendimiento académico de los estudiantes, por tanto, es imprescindible que las universidades pongan especial atención en este rubro, ya que puede hacer la diferencia para retener y mantener acompañado, asesorado y guiado al estudiante hasta terminar sus estudios universitarios. Según Feldman y Theiss (1980) las expectativas de los estudiantes se pueden mantener en términos positivos cuando el docente genera un ambiente de confianza, apertura, acompañamiento e interacción entre estudiantes y el docente.

Derivado de esta investigación existen algunas recomendaciones para futuros estudios. Se sugiere evaluar a otros estudiantes de licenciatura para corroborar si las relaciones entre los constructos abordados permanecen o no. Además, se podría complementar la investigación con un enfoque cualitativo para profundizar sobre las respuestas 
proporcionadas y otras categorías que pudiesen emerger para agregar otras variables que permitan explicar mejor el fenómeno de estudio.

Una limitación de este estudio es que los datos de la muestra se obtuvieron en una universidad, por lo que sus hallazgos no pueden ser generalizables a la realidad de las instituciones de educación superior en la región o en el país.

\section{Referencias}

Aguilera, J.L. (2019). La tutoría universitaria como práctica docente: fundamentos y métodos para el desarrollo de planes de acción tutorial en la universidad. ProPosições, 30, 1-27. http://dx.doi.org/10.1590/19806248-2017-0038

Arbizu, F., Lobato, C., \& del Castillo, L. (2005). Algunos modelos de abordaje de la tutoría universitaria. Revista de Psicodidáctica, 10(1), 7-21. https://www. redalyc.org/pdf/175/17514745002.pdf

Arias, M. L., Arias, E., Arias, J., Ortiz, M., \& Garza, M.G. (2018). Perfil y competencias del docente universitario recomendados por la Unesco y la OCDE. Revista Atlante: Cuadernos de Educación y Desarrollo. https://www.eumed.net/rev/atlante/2018/06/competencias-docente-universitario.html

Armas, M. M. (2019). Hacer fluir el aprendizaje. International Journal of Developmental and Educational Psychology, 2(1), 299-311. https://www.redalyc.org/ jatsRepo/3498/349860126029/html/index.html

Bandura, A. (1977). Self-efficacy: Toward a unifying theory of behavioral change. Advances in Behaviour Research and Therapy, 1(4), 139-161. https://doi. org/10.1016/0146-6402(78)90002-4

Cambours de Donini, A.M., Iglesias, A., \& Muiños de Britos, S.M. (2011). La tutoría en la Universidad: una estrategia para la retención de los estudiantes. Congreso CLABES. http://revistas.utp.ac.pa/index.php/clabes/article/view/853

Campbell, D.T., \& Fiske, D.W. (1959). Convergent and discriminant validation by the multitrait-multimethod matrix. Psychological Bulletin, 56(2), 81-105. https://doi.org/10.1037/h0046016

Castañeda, G., \& Alarcón, G. J. (2019). Evaluación de la satisfacción de alumnos de educación superior del Centro Universitario Sur de la Universidad Autónoma de Tamaulipas. Revista Innovaciones de Negocios, 15(30), 223-241. https://doi.org/10.29105/rinn15.30-5

Conde, A., Deaño, M., Pinto, A.A., Iglesias-Sarmiento, V., Alfonso, S., García-Señorán, M., Limia, S., \& Tellado, F. (2017). Expectativas académicas y planificación. Claves para la interpretación del fracaso y del aban- dono académico. International Journal of Developmental and Educational Psychology, 1(1), 167-176. https://www.redalyc.org/pdf/3498/349852544017. pdf

Cuevas, O., García, R.I., Vales, J.J., \& Cruz, I.R. (2017). Monitoring the results of the Tutoring Program in its face-to-face and virtual modalities on the academic achievement of students at a Mexican university. International Journal of Higher Education, 6(2), 169-181. https://doi.org/10.5430/ijhe.v6n2p169

De Backer, L., Van Keer, H., \& Valcke, M. (2014). Promoting university students' metacognitive regulation through peer learning: the potential of reciprocal peer tutoring. Higher Education, 70(3), 1-18. https:// doi.org/10.1007/s10734-014-9849-3

De la Cruz, G., Chehaybar, E., \& Abreu, L. F. (2011). Tutoría en Educación Superior: Una Revisión Analítica de la Literatura. Revista de la Educación Superior, 4O(157), 189-210. http://publicaciones.anuies.mx/pdfs/revista/Revista157_S5A2ES.pdf

Delgado-García, M., Conde, S., \& Boza, Á. (2020). Perfiles y funciones del tutor universitario y sus efectos sobre las necesidades tutoriales del alumnado. Revista Española de Pedagogía, 78(275), 119-143. https://doi. org/10.22550/REP78-1-2020-03

Díaz, K., \& Osuna, C. (2021). Percepción de los docentes sobre el abandono escolar y su práctica en el aula en bachilleratos tecnológicos de Baja California. Diálogos sobre educación. Temas actuales en investigación educativa, 20(11), 1-17. https://doi.org/10.32870/dse. v0i20.576

Eaton, M.D. (2015). Bridging the Experiential Learning Gap: An Evaluation of the Impacts of Ulster University's Senior Student Tutoring Scheme on First Year Students. Journal of University Teaching and Learning Practice, 12(2), 1-15. https://files.eric.ed.gov/fulltext/ EJ1072382.pdf

Feldman, R.S., \& Theiss, A.J. (1980). The Teacher and Student as Pygmalions: Joint Effects of Teacher and Student Expectations. Journal of Educational Psychology, 74(2), 217-223. https://eric.ed.gov/?q=The+Teacher+and+Student+as+Pygmalions\%3a+Joint+Effects+of+Teacher+and+Student+Expectations.\&ft=on\&id=ED201905

Freixa, M., Llanes, J., \& Venceslao, M. (2018). El abandono en el recorrido formativo del estudiante de ADE de la Universidad de Barcelona. Revista de Investigación Educativa, 36(1), 185-202. https://doi.org/10.6018/ rie.36.1.278971

Guerra-Martín, M.D., Lima-Serrano, M., \& Lima Rodríguez, J.S. (2017). Effectiveness of Tutoring to Improve Academic Performance in Nursing Students at the University of Seville. Journal of new approaches in Educational Research, 6(2), 93-102. https://doi. org/10.7821/naer.2017.7.201

Hernández, R., Fernández, C., \& Baptista, P. (2014). Metodología de la Investigación ( $5^{a}$ ed.). Mc Graw Hill 
Hu, L. \& Bentler, P.M. (2009). Cutoff criteria for fit indexes in covariance structure analysis: Conventional criteria versus new alternatives. Structural Equation Modeling: A Multidisciplinary Journal, 6(1), 1-55. https://doi.org/10.1080/10705519909540118

Instituto Tecnológico de Sonora, ITSON. (2015). Estudio de indicadores de trayectoria escolar, Información de insumo para el taller de análisis del comportamiento de trayectorias escolares.

Kline, R. (2005). Principles and practice of structural equation modelling (2a ed.). The Guilford Press.

Lobato, C., Arbizu, F., \& del Castillo, L. (2004). Las representaciones de la tutoría universitaria en profesores y estudiantes: estudio de un caso. Educación XXI, (7), 135-168. https://www.redalyc.org/ pdf/706/70600707.pdf

Mares, G., Rocha, H., Rivas, O., Rueda, E., Cabrera, R., Tovar, J., \& Medina, L., (2012). Identificación de factores vinculados con la deserción y la trayectoria académica de los estudiantes de Psicología en la Fes Iztacala. Enseñanza e Investigación en Psicología, 17(1), 189-207. https://www.redalyc.org/articulo. oa?id=29223246012

Martínez, L.M., Conejo, F., \& Rodríguez, A.V. (2017). La acción tutorial como experiencia educativa para la formación integral de los estudiantes de Medicina. Revista Actualidades Investigativas en Educación, 17(3), 1-23. http://dx.doi.org/10.15517/aie.v17i3.30099

Martínez, P., Martínez, M., \& Pérez, J. (2016). ¿Cómo avanzar en la tutoría universitaria? Estrategias de acción: Los estudiantes tienen la palabra. Revista Española de Orientación y Psicopedagogía, 27(2), 80-98. https://www.redalyc.org/articulo. oa?id=338246883006

Morgan, M. (2015). Study expectations of different domiciled Postgraduate-Taught students in a UK post-1992-institution. Quality Assurance in Education, 23(3), 233-249. https://doi.org/10.1108/QAE-022014-0005

Naranjo, M.L. (2010). Motivación: Perspectivas teóricas y algunas consideraciones de su importancia en el ámbito educativo. Revista Educación, 33(2), 153-170. http://www.redalyc.org/articulo. oa?id $=44012058010$

Nunnally, J., \& Bernstein, I. (1995). Teoría Psicométrica (3a . ed.). McGraw-Hill.

Organización de las Naciones Unidas para la Educación, la Ciencia y la Cultura, \& Organización Internacional del Trabajo (2008), Ginebra, Suiza: UNESCO. OIT. https://www.snte.org.mx/pdf/RecomendacionesOITyUNESCO.pdf

Orozco, E. E., Nájera, J.F., Guerra, S. E., Ramos, F., \& Guerra, R. M. (2021). Reflexión sobre las competencias docentes en los institutos superiores tecnológicos en Ecuador. Educación Médica Superior, 35(1). http:// scielo.sld.cu/scielo.php?script=sci_arttext $\&$ pi$\mathrm{d}=$ S086421412021000100020\&lng=es\&tlng=es.
Pérez, F. J., González, C., González, N., \& Martínez, M. (2017). Tutoría en la Universidad: un estudio de caso en la Facultad de Educación en la Universidad de Murcia. Educatio Siglo XXI, 35(2), 91-110. https://doi. org $/ 10.6018 / \mathrm{j} / 298531$

Pérez, J. (2015). Expectativas, satisfacción y rendimiento académico en alumnado universitario. Revista de Psicología y Educación, 10(1), 11-32. http://www.revistadepsicologiayeducacion.es/pdf/115.pdf

Pichardo, M. C., García, A. B., De la Fuente, J., \& Justicia, F. (2007). El estudio de las expectativas en la universidad: análisis de trabajos empíricos y futuras líneas de investigación. Revista Electrónica de Investigación Educativa, 9(1), 1-16. http://www.scielo.org. $\mathrm{mx} / \mathrm{pdf} / \mathrm{redie} / \mathrm{v} 9 \mathrm{n} 1 / \mathrm{v} 9 \mathrm{n} 1 \mathrm{a} 1 . \mathrm{pdf}$

Ramírez, M. R., \& Olmos, H.I. (2020). Funciones cognitivas y motivación en el aprendizaje de las matemáticas. Naturaleza y Tecnología, (2), 51-63. http://www.naturalezaytecnologia.com/index.php/nyt/article/ view/383/289

Reeve, J. (2008). Understanding motivation and emotion (5a ed.). Harcourt Brace College Publishers

Rodríguez-Pérez, N. (2012). Causas que intervienen en la motivación del alumno en la enseñanza aprendizaje de idiomas: el pensamiento del profesor. Didáctica, Lengua y Literatura, 24, 381-409. https://doi. org/10.5209/rev_DIDA.2012.v24.39932

Román, M. (2013). Factores asociados al abandono y la deserción en América Latina: Una mirada en conjunto. REICE. Revista Iberoamericana sobre Calidad, Eficacia y Cambio en Educación, 11(2), 33-59. https:// www.redalyc.org/pdf/551/55127024002.pdf

Salazar, C.M., Chiang, M.M., \& Muñoz, Y.A. (2016). Competencias docentes en la educación superior: Un estudio empírico en la Universidad del Bío Bío. Revista Electrónica Actualidades Investigativas en Educación, 6(1), 1-28. http://dx.doi.org/10.15517/aie.v16i1.22383

Satorra, A., \& Bentler, P. M. (2001). A scaled difference chi-square test statistic for moment structure analysis. Psychometrika, 66(1), 507-514. https://doi. org/10.1007/BF02296192

Stover, J.B., Bruno, F.E, Uriel, F., \& Fernández, M. (2017). Teoría de la autodeterminación: Una revisión teórica. Perspectivas en Psicología: Revista de Psicología y Ciencias Afines, 14(2), 105 - 115. https://www.redalyc.org/pdf/4835/483555396010.pdf

Torres, A.D., Badillo, M., Valentin, N.O., \& Ramírez, E.T. (2014). Las competencias docentes: el desafío de la educación superior. Innovación Educativa, 14(66), 129-145. http://www.scielo.org.mx/pdf/ie/v14n66/ v14n66a8.pdf

Torres, M. (2016). Factores de desmotivación más sobresalientes en el aprendizaje: La afectividad requisito ineludible. Investigación Cualitativa en Educación, 1, 732-735. https://proceedings.ciaiq.org/index.php/ ciaiq2016/article/view/663

Triana, M.L., Jadán, P. Y., Sánchez, M.S., Defaz, G.J., \& García, 
R.E. (2016). Papel de la motivación docente en el desarrollo de conductas positivas de los estudiantes de las carreras de Idiomas y Secretariado Bilingüe de la Universidad Técnica de Babahoyo, Extensión Quevedo, Ecuador. Didasc@lia: Didáctica y Educación, 7(6), 329-338. https://dialnet.unirioja.es/descarga/articulo/6672974.pdf

Uzuner, S., \& Aktas, E. (2016). The effects of peer tutoring on university students success, speaking skills and speech self-efficacy in the effective and good speech course. Educational Research and Reviews, 11(11), 1035-1042. https://academicjournals.org/ journal/ERR/article-full-text-pdf/1936FBF58886

Villalobos, A., Melo, Y., \& Pérez, C. (2010). Percepción y expectativas de los alumnos universitarios frente al profesor no pedagogo. Estudios Pedagógicos, 36(2), 241-249. https://doi.org/10.4067/S071807052010000200014

Walvoord, M.E., \& Pleitz, J.D. (2016). Applying Matched Sampling to Evaluate a University Tutoring Pro- gram for First-Year Students. Learning Assistance Review, 21(1), 99-113. https://files.eric.ed.gov/fulltext/EJ1095718.pdf

West, S. G., Finch, J. F., \& Curran, P. J. (1995). Structural equation modelling with non normal variables: problems and remedies. En R. H. Hoyle (Ed.), Structural equation modeling. Concepts, issues, and applications (pp. 56-75). Sage Publications Inc.

Zavala, M., González, I., Rodríguez, A., \& Vázquez, M. (2015). Factores asociados a la deserción escolar. Estudio exploratorio basado en la experiencia del desertor. En R. Pérez, A. Rodríguez \& E. Álvarez (Eds.), Innovación en la educación superior. Desafíos y propuestas (pp. 297-301). Ediciones de la Universidad de Oviedo.

Zavala, M.A., Álvarez, M., Vázquez, M.A., González, I., \& Bazán, A. (2018). Factores internos, externos y bilaterales asociados con la deserción en estudiantes universitarios. Interacciones, 4(1), 59-69. https://doi. org/10.24016/2018.v4n1.103

RIDU / Revista Digital de Investigación en Docencia Universitaria / e-ISSN: 2223-2516

(C) Los autores. Este artículo es publicado por la Revista Digital de Investigación en Docencia Universitaria del Área de Institutional Research and Effectiveness de la Dirección de Aseguramiento de la Calidad, Universidad Peruana de Ciencias Aplicadas. . "Este es un artículo de acceso abierto, distribuido bajo los términos de la LicenciaCreativeCommons Atribución 4.0 Internacional (CC BY 4.0) (https://creativecommons.org/licenses/by/4.0/deed.es), que permite el uso, distribución y reproducción en cualquier medio, siempre que la obra original sea debidamente citada." 